\title{
Ranking and selection of commercial off-the-shelf using fuzzy distance based approach
}

\author{
Rakesh Garg $^{\mathrm{a}, \mathrm{b}}$, R. K. Sharma ${ }^{\mathrm{a}^{*}}$ and Kapil Sharma ${ }^{\mathrm{b}}$
}

${ }^{a}$ Department of Computer Science \& Engineering, Al-Falah School of Engineering \& Technology, Faridabad, Haryana, India ${ }^{b}$ Department of Computer Science and Engineering, Delhi Technological University, Delhi, India

\section{H R O N I C L E}

Article history:

Received June 25, 2015

Received in revised format:

October 12, 2015

Accepted December 15, 2015

Available online

December 172015

Keywords:

Commercial Off-the-Shelf (COTS)

Fuzzy Distance Based Approach

(FDBA)

Selection Criteria

CBSE

\begin{abstract}
A B S T R A C T
There is a tremendous growth of the use of the component based software engineering (CBSE) approach for the development of software systems. The selection of the best suited COTS components which fulfils the necessary requirement for the development of software(s) has become a major challenge for the software developers. The complexity of the optimal selection problem increases with an increase in alternative potential COTS components and the corresponding selection criteria. In this research paper, the problem of ranking and selection of Data Base Management Systems (DBMS) components is modeled as a multi-criteria decision making problem. A 'Fuzzy Distance Based Approach (FDBA)' method is proposed for the optimal ranking and selection of DBMS COTS components of an e-payment system based on 14 selection criteria grouped under three major categories i.e. 'Vendor Capabilities', 'Business Issues' and 'Cost'. The results of this method are compared with other Analytical Hierarchy Process (AHP) which is termed as a typical multi-criteria decision making approach. The proposed methodology is explained with an illustrated example.
\end{abstract}

(C) 2016 Growing Science Ltd. All rights reserved.

\section{Introduction}

It was the late 70's when the concept of reusable components was introduced in structured programming by the software developers to maintain and develop software systems and it led the development of object oriented software systems in 80's. However, it is today's growing complexity of systems that forces us to turn this idea into practice. Component-Based Software Engineering (CBSE) is concerned with designing, selecting and composing components (Crnkovic \& Larsson, 2002). CBSE is emerged as new approach to solve the development of complex problem. CBSE is concerned with the assembly of off the shelf components using well defined system architecture. CBSE is about building software through composition. The main purpose of using CBSE is that it allows reusability for selecting the components, adapting the components and then integrating them using a pre-defined architecture. A component once developed can be used further to develop new software systems which * Corresponding author. Tel: 91-9992995488

E-mail address: rkgarg06@gmail.com (R. K. Sharma)

(C) 2016 Growing Science Ltd. All rights reserved. doi: $10.5267 /$ j.dsl.2015.12.004 
will result in the less development effort. The main success factor while implementing the CBSE approach is the component selection that mainly depends on the optimization of (i) software requirements satisfaction and (ii) development cost.

The component-based software engineering emphasises mainly on the integration of existing COTS component into the target system. So the component selection and their integration become the two main issues, which play a vital role in the growth and development of the component based systems. Component selection is very important factor in component selection problem because there is a chance that more than one component satisfy the target requirements. The use of COTS component leads to the better software quality and reduces development time and the cost. The development of componentbased software needs reuse of the components which interacts mutually as well as with the architecture of the system. No standard method exists in the past to describe, define and compose these COTS components or third party components in the final system. The development of the software which is based on COTS is one demanding process that offers the potential reuse of existing components with the reduction of software complexity (Tran et al., 1997). Due to a number of major benefits such as better software quality, less development effort etc. provided by the COTS based development approach has become more and more popular in the IT sector (Kontio, 1996; Alves \& Castro, 2001; Phillips \& Polen, 2002; Ncube \& Maiden, 1999; Ibrahim et al., 2007; Erdogmus, 2001). However, development of software based on COTS is a nontrivial task. Such tasks are associated with major challenges e.g. uncertainty, information lack, standards etc.; (Gashi et al., 2007; Mohamed et al., 2007; Ziv et al., 1997; Mead, 2008; Ibrahim et al., 2008, 2009). The software developed by adopting the component based Software engineering approach is generally the integration of commercial off the shelf (COTS) components provided by various vendors that fulfill the desired technical and nontechnical requirements of the target system. In the COTS- based software development, the complicated system is decomposed into the various that support the identification of multiple COTS components for evaluation. Although, the use of the COTS component lead to the great benefits such as time and cot development, there arises the problem of the COTS evaluation and selection due to the lack of information, high uncertainty etc.

The paper is organized as follows: in Section 2, the component selection problem and multi criteria decision making analysis are described. A review of the existing COTS selection methods is provided in Section 3. The proposed methodology for COTS component selection is provided in Section 4. A case study on E-payment system is provided in Section 5 to show the applicability of proposed methodology. The results and comparison with other existing methodologies are analyzed in Section 6 and Section 7 contains the conclusion of the methodology presented in this paper.

\section{Problem Formulation and Research Motivation}

The primary focus of the CBSE approach is on the integration of various COTS components to produce the target system or software. The major problem in CBSE approach is the selection and integration of components in the final target system such that the final system satisfy all the requirements which were stated at earlier stages of the software development. If a set of components is provided then the component selection problem may be categorized into two main selection problems based on the objectives and the criteria for selection. These two categories are shown in Fig. 1.

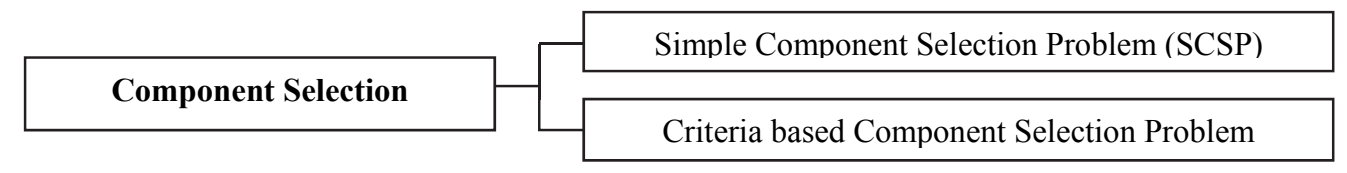

Fig. 1. Classification of the problem of component selection

\subsection{Simple Component Selection problem (SCSP)}

The problem, which involves the selection of a subgroup of software components among a given large group of such software components or from repository in such a manner that the chosen subset of 
software components is capable enough to satisfy the predetermined set of objectives of the problem is defined as Simple Component Selection Problem (SCSP).

\subsection{Criteria Based Component Selection problem (CCSP)}

Criteria-based Component Selection Problem (CCSP) may be defined as the problem of selection of a subgroup of software components among a given large group of such software components or from repository in a way that the composition of the chosen subset of software components fulfils the set of objectives of the problem and using various criteria.

The schematic selection procedure of components based on multiple criteria is given in Fig. 2 .

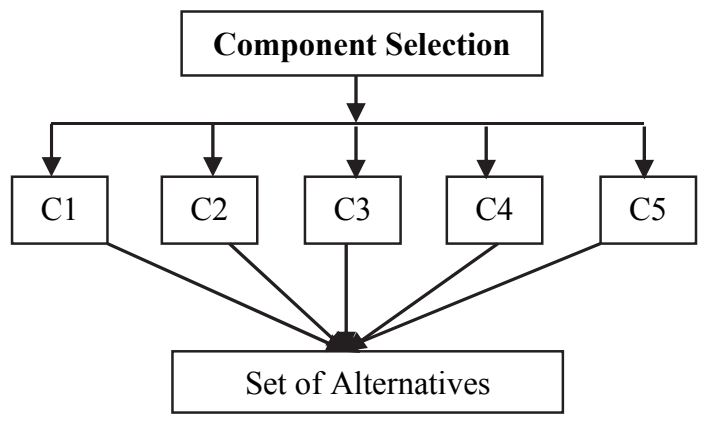

Fig. 2. Criteria based component selection problem

\subsection{Multi Criteria Decision Analysis (MCDA)}

A Multi Criteria Decision Analysis (MCDA) method helps the decision makers to select the optimal or best suitable alternative solution or component among a large number of potentially viable alternative solutions or components. The selection is based on particular requirements and ratings of these alternatives based on criteria to be taken under consideration. The basic activities involved in MCDA are as follows:

1. Establishment of various criteria that the selected component should fulfill.

2. Assigning a weight to each to represent the relative importance.

3. Evaluating the fitness of each component against defined selection.

4. Finally, Rank the components based on how well they fit the criteria.

\section{Previous Related Work}

Kontio (1995) developed a method of OTSO for the selection and evaluation of the components based on the product quality characteristics. Finkelstein et al. (1996) addressed the problem of the requirement specficataion and their deployment in an accuurate manner whereas. Wong and Jeffery (1996) proposed a method based on the gutman's means-end chain model for evaluation of software quality by considering the importnace of non ISO-9126 chracterstics in the software quality evaluation. Gaedke et al. (1999) proposed web components repository as a tool that supports the reuse of code of existing component to develop a new system. The repository introduced by the researchers in this study mainly consists of three parts as (i) component store (ii) metadata store and (iii) search tool.

Ncube and Dean (2002) compared various existing approaches for the component selection and developed a more effective approach based on requirement-driven. Phillips and Polen (2002) provided an approach for COTS selection that mainly stresses on the creation and maintenance of a data repository that can help the developers select the appropriate component for the software development. Rus et al. (2001) built the web based repository by extracting information from research articles published in journal, conference proceedings and project reports. Morisio et al. $(2000,2002)$ provided a new process and the proper guidelines for the selection and evaluation of COTS components by identifying the main differences as requirements definition, high level design, testing etc. in comparison to the traditional approach for the software development. 
Ruhe (2003) addressed the COTS selection problem with a high uncertainty and lack of information and developed a software namely COTS-DSS for the selection and evaluation of COTS. Kunda (2003) presents an approach incoroporating the non technical isues associated in the selection of COTS components and is named as social technical approach. Ducik and Boegh (2003) present a framework that helps the software developer in the quality evaluation process of the COTS software product. Mohamed et al. (2004) provide a conceptual model for the component selection and evaluation that was implemented by using the agent technologies incorporated by toe knowledge bases as COTS KB and methods KB. Mancebo and Andrews (2005) present a component selection and evaluation approach based on the existing techniques by considering the archietecture of the components as a major aspect. Mohamed et al. (2005) proposed a two level mainly process level and activity level customization process for the selection process by considering the attributes related to the project domain such as project effort. In the contemporary work, Mohamed et al. (2007) addressed the problem of COTS selection and evaluation and provide a survey on the contribution of the most significant eighteen existing COTS selection approaches provided by various researchers.

Gesellensetter and Glesner (2007) developed a unified approach for the development of component based systems using methods from code optimization which further helps the software developer to select the optimal components for the target system. Haghpanah et al. (2007) presented the problem of component selection and evaluation as NP-complete problem and proposed a method based on the greedy approach and the genetic algorithm by taking the computational complexity of this problem into account.

Saltelli et al. (2008) introduced the concept of sensitivity analysis which provides the basis for obtaining the useful information about the selection parameters which can contribute up to a large extent to the uncertainties in the output of the model. Ibrahim et al. (2009) present a hybrid approach for the selection and evaluation of COTS components by combining two approaches as analytical hierarchy processing and the bayesian-belief network and the selection criteria uncertainty was considered in this research.

Mittal and Bhatia (2013) considered reusability as a major selection criterion for the component selection and solved the problem of COTS component selection and evaluation by applying analytic hierarchy processing technique. Faridi et al. (2013) presented the characteristics described in the software quality codes for the component selection and evaluation. Faundes et al. (2013) proposed a method process based evaluation and comparison for the selection of OTSS by accounting six criteria as Coverage, Automation, Implementation, Cost, Collaboration, and Participation.

Kaur and Singh (2014) solved the problem of component selection and evaluation by applying Promethee method and they considered performance, reliability, maintainability, cost and inerrability as COTS selection criteria. Nazir et al. (2014) presented effectiveness, efficiency, satisfaction, safety and usability as the major selection criteria for the COTS component selection and evaluation. Vinay et al. (2014) developed a W-shaped framework for component selection by considering the problem of component selection and evaluation problem as NP-complete problem due to the high availability of the selection parameters in literature. As the reusability approach in the software development leads to the great benefits such as reduction in cost and time, a framework based on the component based software engineering for the reusability was developed (Khan et al., 2014).

\section{Proposed Methodology}

Fuzzy Distance Based Approach (FDBA) is used for ranking and evaluation of COTS components for the first time. Fuzzy set theory is used for assigning weights to the selection criteria and performance ratings of the COTS components. Fuzzy operators are used to determine average aggregated weights and performance ratings. Distance Based Approach is further applied to determine the composite distance of the alternative COTS components from the optima, which is an imaginary component having all ideal values for each selection criteria. The ranking of the COTS components is done on the ascending/descending order of the values of the composite distance of the COTS components from the optimal value i.e. zero. The DBA has already been applied for the evaluation, selection and ranking of 
robots and software engineering metrics, etc. (Kumar and Garg, 2010). The composite distance value is:

$$
C D_{O P-A l t}=\left[\sum_{j=1}^{m}\left(Z_{O P j}-Z_{i j}\right)^{2}\right]^{1 / 2}
$$

where $Z_{i j}=\frac{r_{i j}-\bar{r}_{j}}{S_{j}}, \bar{r}_{j}=\frac{1}{n} \sum_{i=1}^{n} r_{i j} ; S_{j}=\left[\frac{1}{n} \sum_{i=1}^{n}\left(r_{i j}-\bar{r}_{j}\right)^{2}\right]^{1 / 2}$

$[r]=\left[\begin{array}{cccc}r_{11} & r_{12} & \ldots & r_{1 j} \\ r_{21} & r_{22} & \ldots & r_{2 j} \\ \vdots & \vdots & \vdots & \vdots \\ r_{i 1} & r_{i 2} & \ldots & r_{i j} \\ r_{b 1} & r_{b 2} & \cdots & r_{b j}\end{array}\right] ;$

Here, $i, j$ represent COTS components and selection criteria, respectively. $r_{b j}$ is the best (optimal) value for a particular selection criteria among all alternate COTS components.

\section{An Empirical Study}

In the past decades, as the software development increases rapidly, management of data has become a very crucial issue. Generally for the purpose of management of data software developers use the concept of Databases. The power of the databases comes from a body of knowledge and technology that has developed and is embodied in the software applications known as database management systems (DBMS). Database management systems (DBMS) are computer software applications that make interaction with the user, other applications and the database itself to capture and analyze data.

In this study, a group of ten experts from various software companies according to their vast knowledge in this field was established. As the data that can contribute a lot to the COTS selection and evaluation present in the open literature is not sufficient due to lack of maturity, expert's opinion approach was adopted in this research to collect the data that is relevant to the COTS selection and evaluation problem. Firstly, a team of experts from academia as well as IT companies having wide experience in the area of component based software engineering was formed. The experts search for DBMS COTS candidates and finally, provide a number of DBMS candidates as COTS (i) Oracle (ii) SQL-Express (iii) MYSQL (iv) Ms-Access (v) Postgreys. The DBMS COTS components identified by experts can be compared by the mean of (i) Quality Characteristics (ii) Technology factors (iii) Domain and Architectural Factors (iv) Strategic Factors, named as ranking criteria. The main focus in this study is on strategic factors as shown in Fig. 3.

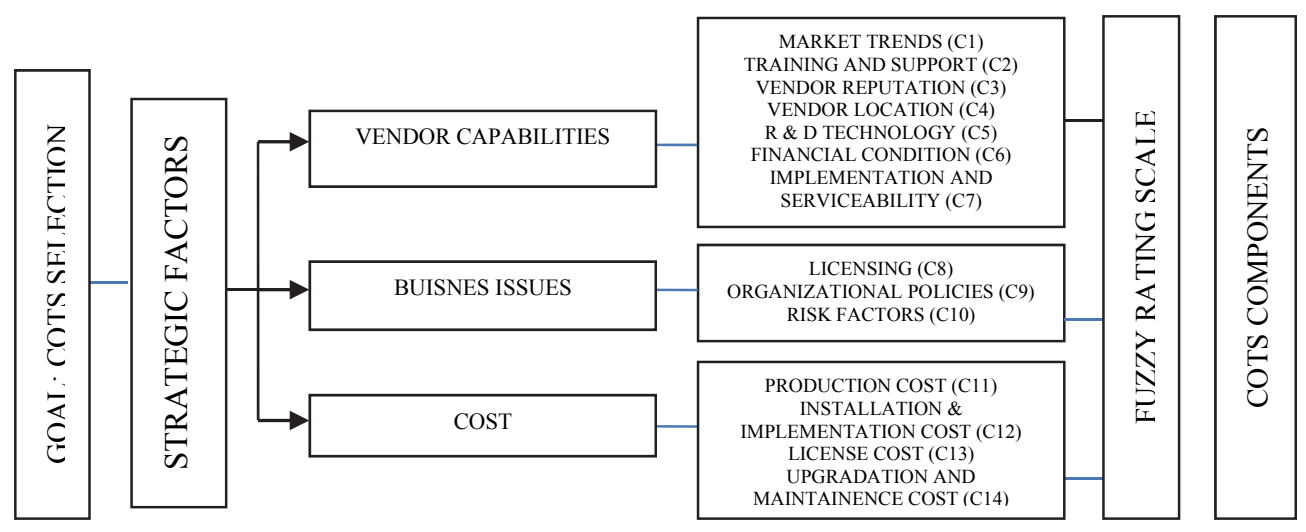

Fig. 3. COTS ranking criteria

The main motive of this research is to design a framework that provides the functionality to rank the various COTS components on the basis of various relevant COTS selection criteria/sub-criteria. An example of the selection of COTS components of a Data Base management System for an E-payment 
system has been taken for ranking of five COTS components based on 14 selection criteria grouped in three broad categories namely 'Vendor capabilities', 'business issues' and 'cost' which fall under the category of Strategic Factors for the selection of COTS. The example will demonstrate and validate the proposed method. The present research is exploratory in nature. Questionnaire is considered to be the best way of collection of data in survey researches which are exploratory in nature. Since, no secondary data is available in the open literature and hence questionnaires are used to collect primary data. Two questionnaires were prepared. The first questionnaire is related to estimate the weights of the COTS selection criteria and the second questionnaire is used to get the performance ratings of the COTS components based on each selection criteria. The weights and ratings are obtained on a seven point fuzzy scale. The questionnaires are designed in three parts: First part of each questionnaire contains a covering letter which explains the purpose of the research study and statement of confidentiality. Second part consists of demographic details e.g. name of the organization, field of expertise (software development, software design, software purchasing and procurement, etc.); length of experience, qualification and designation, etc. Third part of the questionnaire consists of assigning weights to the selection criteria/performance ratings of the COTS components on a predetermined seven point fuzzy scale. The weights (Local and Global) of the selection criteria using the expert opinion are estimated and given in Table 1.

Table 1

Weights (Local and Global) for ranking criteria

\begin{tabular}{clccclcc}
$\begin{array}{c}\text { S. } \\
\text { No. }\end{array}$ & Criterion/Sub-Criterion & $\begin{array}{c}\text { Local } \\
\text { Wt. }\end{array}$ & $\begin{array}{c}\text { Global } \\
\text { Wt. }\end{array}$ & $\begin{array}{c}\text { S. } \\
\text { No. }\end{array}$ & Criterion/Sub-Criterion & $\begin{array}{c}\text { Local } \\
\text { Wt. }\end{array}$ & $\begin{array}{c}\text { Global } \\
\text { Wt. }\end{array}$ \\
\hline Vendor Capabilities & - & 0.4415 & Business Issues & - & 0.2605 \\
\hline C1 & Market Trends & 0.1367 & 0.06035 & C8 & Licensing Arrangements & 0.2967 & 0.0772 \\
C2 & Training and Support & 0.1415 & 0.06247 & C9 & Organizational Policies & 0.3496 & 0.0910 \\
C3 & Vendor Reputation & 0.1319 & 0.05823 & C10 & Risk factors & 0.3837 & 0.0999 \\
C4 & Vendor Location & 0.1319 & 0.05823 & Cost & & - & 0.2980 \\
C5 & R \& D Technology & 0.1127 & 0.04975 & C11 & Production Cost & 0.2931 & 0.0873 \\
C6 & Financial Condition & 0.1751 & 0.07730 & C12 & Installation \& Implementation Cost & 0.2522 & 0.0751 \\
C7 & Implementation \& Serviceability & 0.1703 & 0.07518 & C13 & License Cost & 0.1954 & 0.0582 \\
& & & & C14 & Upgradation \& Maintenance Cost & 0.2593 & 0.0772 \\
\hline
\end{tabular}

A summary of the aggregated average performance ratings assigned by the experts for each criterion is presented in Table 2 .

\section{Table 2}

Aggregated average performance ratings of DBMS COTS components

\begin{tabular}{|llllllll}
\hline Criterion/ Sub-Criterion & Oracle & SQL Express & MYSQL & MS-Access & Postgreys \\
\hline Strategic Factors & 0.3864 & 0.5079 & 0.7960 & 0.4246 & 0.7600 \\
Vendor Capability & 0.4675 & 0.6042 & 0.7935 & 0.4117 & 0.7928 \\
\hline C1 $\quad$ Market Trends & 0.5909 & 0.6091 & 0.8363 & 0.3727 & 0.8182 \\
C2 & Training and Support & 0.2500 & 0.5545 & 0.8500 & 0.3546 & 0.8091 \\
C3 $\quad$ Vendor Reputation & 0.7000 & 0.7727 & 0.7727 & 0.4636 & 0.7363 \\
C4 $\quad$ Vendor Location & 0.3364 & 0.6818 & 0.6273 & 0.5000 & 0.6454 \\
C5 $\quad$ R \& D Technology & 0.5909 & 0.7363 & 0.8091 & 0.3182 & 0.8682 \\
C6 $\quad$ Financial Condition & 0.2318 & 0.4636 & 0.8863 & 0.4091 & 0.8818 \\
C7 $\quad$ Implementation and Serviceability & 0.5727 & 0.6636 & 0.7727 & 0.4636 & 0.7909 \\
\hline Business Issues & 0.4349 & 0.4879 & 0.6879 & 0.4758 & 0.5848 \\
\hline C8 $\quad$ Licensing Arrangements & 0.3364 & 0.4455 & 0.7909 & 0.4091 & 0.7909 \\
C9 $\quad$ Organizational Policies & 0.2318 & 0.4636 & 0.5364 & 0.3909 & 0.3546 \\
C10 $\quad$ Risk Factors & 0.7363 & 0.5545 & 0.7363 & 0.6273 & 0.6091 \\
\hline Cost & & 0.2568 & 0.3955 & 0.9068 & 0.3864 & 0.9023 \\
\hline C11 $\quad$ Production cost & 0.2818 & 0.4818 & 0.9409 & 0.3909 & 0.9727 \\
C12 $\quad$ Installation and Implementation cost & 0.2273 & 0.3727 & 0.9318 & 0.3364 & 0.8682 \\
C13 $\quad$ License Cost & 0.2864 & 0.3364 & 0.9318 & 0.4091 & 0.9591 \\
C14 $\quad$ Upgradation and Maintenance Cost & 0.2318 & 0.3909 & 0.8227 & 0.4091 & 0.8091 \\
\hline
\end{tabular}

The adjusted matrix so formed can be given as: 


$$
\left[r_{a}\right]=\left[\begin{array}{rrrrrrr}
0.2182 & 0 & 0.2364 & 0 & 0.2727 & 0 & 0.1091 \\
0.2364 & 0.3045 & 0.3091 & 0.3454 & 0.4181 & 0.2318 & 0.2 \\
0.4636 & 0.6 & 0.3091 & 0.2909 & 0.4909 & 0.6545 & 0.3091 \\
0 & 0.1046 & 0 & 0.1636 & 0 & 0.1773 & 0 \\
0.4455 & 0.5591 & 0.2727 & 0.309 & 0.55 & 0.65 & 0.3273 \\
0.4636 & 0.6 & 0.3091 & 0.3454 & 0.55 & 0.6545 & 0.3273
\end{array}\right]
$$

The average and standard deviation are $0.27274,0.31364,0.22546,0.22178,0.459867,0.34634$, 0.1891 and $0.190365,0.266648,0.129591,0.141632,0.219601,0.295276,0.137638$ respectively. By using the Z-formula, the standardized matrix is obtainted.

Now, the distance matrix can be represented as:

$$
\left[r_{d}\right]=\left[\begin{array}{rrrrrrr}
1.2891 & 2.2502 & 0.5610 & 2.4387 & 1.2627 & 2.2166 & 1.5853 \\
1.1935 & 1.1082 & 0 & 0 & 0.6006 & 1.4315 & 0.9249 \\
0 & 0 & 0 & 0.3848 & 0.2691 & 0 & 0.1322 \\
2.4353 & 1.8579 & 2.3852 & 1.2836 & 2.5045 & 1.6161 & 2.3707 \\
0.0951 & 0.1534 & 0.2809 & 0.2570 & 0 & 0.0152 & 0
\end{array}\right]
$$

Finally the resultant matrix having composite distance will be given as:

$$
\left[r_{c}\right]=\left[\begin{array}{rrrrrrr}
1.6618 & 5.0632 & 0.3147 & 5.9474 & 1.5945 & 4.9132 & 2.5132 \\
1.4244 & 1.2281 & 0 & 0 & 0.3608 & 2.0493 & 0.8554 \\
0 & 0 & 0 & 0.1481 & 0.0724 & 0 & 0.0175 \\
5.9308 & 3.4517 & 5.6892 & 1.6477 & 6.2727 & 2.6118 & 5.6548 \\
0.0090 & 0.0235 & 0.0789 & 0.0661 & 0 & 0.0002 & 0
\end{array}\right]
$$

By using Eq. (1) it can be seen in Table 3 that the proposed methodology for COTS selection uses the basis of distance of each alternative COTS from the best or optimum hypothetical solution for quantitative comparison and ranking of COTS. This distance is termed as composite distance and overall ranking of COTS based on this composite distance value is shown in Table 3.

Table 3

Overall Ranking of DBMS COTS Component using FDBA

\begin{tabular}{lcccccccc}
\hline \multirow{2}{*}{$\begin{array}{l}\text { COTS } \\
\text { Components }\end{array}$} & \multicolumn{2}{c}{ Vendor Capabilities } & \multicolumn{2}{c}{ Business Issues } & \multicolumn{2}{c}{ Cost } & \multicolumn{2}{c}{ Strategic Concerns } \\
\cline { 2 - 9 } & Composite Distance & Rank & $\begin{array}{c}\text { Composite } \\
\text { Distance }\end{array}$ & Rank & $\begin{array}{c}\text { Composite } \\
\text { Distance }\end{array}$ & Rank & $\begin{array}{c}\text { Composite } \\
\text { Distance }\end{array}$ & $\begin{array}{c}\text { Rank } \\
\text { ORACLE }\end{array}$ \\
SQL EXPRESS & 2.69127 & 4 & 3.36158 & 5 & 4.337614 & 5 & 3.725443 & 5 \\
MYSQL & 2.432702 & 3 & 2.816597 & 4 & 3.477869 & 3 & 2.700875 & 3 \\
MS-ACCESS & 0.487837 & 2 & 0 & 1 & 0.4568 & 2 & 0 & 1 \\
POSTGREYS & 5.590946 & 5 & 2.538834 & 3 & 3.528409 & 4 & 3.423775 & 4 \\
\hline
\end{tabular}

In order to validate the methodology and the results obtained, the present COTS component selection problem using the same datasets is solved using AHP. The results so obtained are given in Table 4 . The comparison shows that the results obtained using FDBA method and AHP are same for the selection criteria - cost and strategic concerns. In case of selection criteria namely 'Vendor Capabilities' and 'Business Issues' a minor differences in the rankings of two COTS components is observed. In case of AHP method MYSQL is ranked at \#1 followed by POSTGREYS whereas the POSTGREYS is ranked 
at \#1 followed by MYSQL using FDBA method. The reason is quite obvious that there is a negligible difference in the ranking values (0.7971 and 0.7954) of these two components using AHP. Same pattern is observed in the ranking values of SQL EXPRESS and MS-ACCESS components for selection criterion namely 'Business Issues'. It is thus evident that the FDBA method is validated.

Table 4

Overall Ranking of DBMS COTS Component using AHP

\begin{tabular}{|c|c|c|c|c|c|c|c|c|}
\hline \multirow{2}{*}{$\begin{array}{l}\text { COTS } \\
\text { Components }\end{array}$} & \multicolumn{2}{|c|}{ Vendor Capabilities } & \multicolumn{2}{|c|}{ Business Issues } & \multicolumn{2}{|c|}{ Cost } & \multicolumn{2}{|c|}{ Strategic Concerns } \\
\hline & Composite Distance & Rank & $\begin{array}{c}\text { Composite } \\
\text { Distance }\end{array}$ & Rank & $\begin{array}{c}\text { Composite } \\
\text { Distance }\end{array}$ & Rank & $\begin{array}{c}\text { Composite } \\
\text { Distance }\end{array}$ & Rank \\
\hline ORACLE & 0.4575 & 4 & 0.4412 & 5 & 0.2559 & 5 & 0.3962 & 5 \\
\hline SQL EXPRESS & 0.6306 & 3 & 0.4903 & 3 & 0.4023 & 3 & 0.5276 & 3 \\
\hline MYSQL & 0.7971 & 1 & 0.6826 & 1 & 0.9061 & 2 & 0.7997 & 1 \\
\hline MS-ACCESS & 0.4146 & 5 & 0.4799 & 4 & 0.3854 & 4 & 0.4208 & 4 \\
\hline POSTGREYS & 0.7954 & 2 & 0.5740 & 2 & 0.9401 & 1 & 0.7712 & 2 \\
\hline
\end{tabular}

\section{Results}

According to methodology adopted in this empirical study, lower value of composite distance implies better ranking. The comparative rankings of all five COTS components based on vendor capabilities, Business issues and cost individually as well as taken altogether are given in Fig. 4. It is depicted that Postgreys has been ranked first on the basis of vendor capabilities and cost as selection criteria. MYSQL and SQL Express are ranked second and third, respectively and fall among top three DBMS cots components. Oracle is ranked at five based on Business issues, cost and Strategic factors whereas ranked at four based on vendor capabilities which shows the least use of this component. MYSQL is ranked at \#1 based on strategic concern i.e. considering all three selection criteria namely vendor capabilities, Business issues and cost collectively, and is followed by Postgreys whereas Oracle is ranked at last.

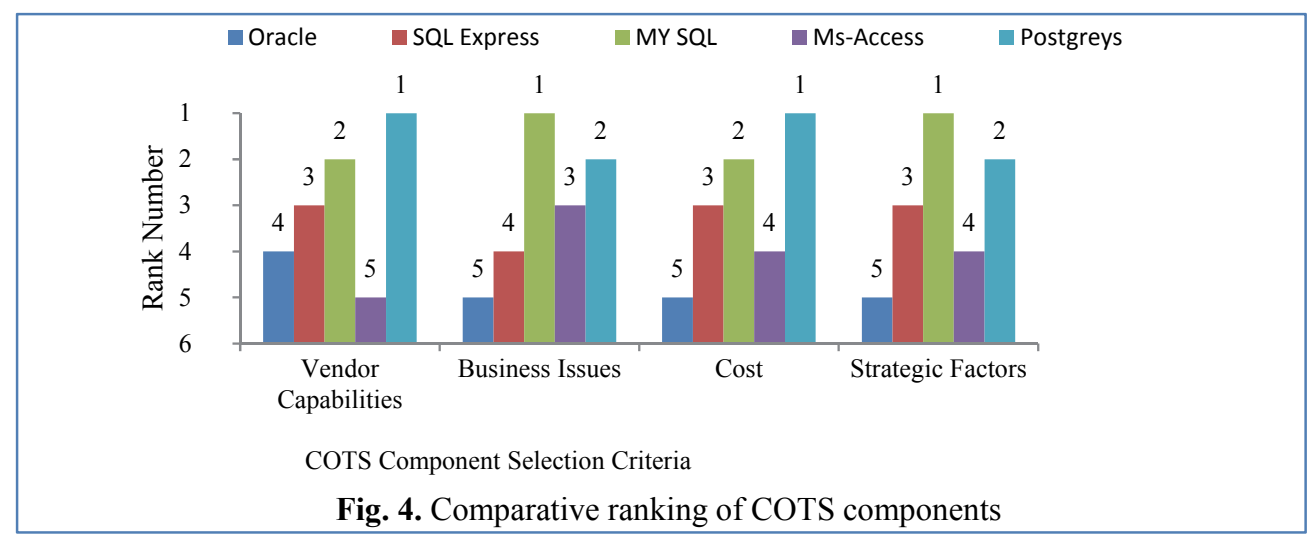

\section{Conclusions}

The aim of the research paper was to resolve the major problems of optimal selection of COTS components for DBMS of an e-payment system while implementing the concept of component based system development. The problem of ranking and selection of COTS components of database management system is modeled as a multi-criteria decision making problem. The selection component problem relates to an e-payment system. The alternative potential COTS components are ranked based on a number of mutually conflicting selection criteria using 'Fuzzy Distance Based Approach'. The developed approach had not been employed earlier and can be termed as a benchmark concept for the rapid solution of such type of component selection problems. The proposed 'Fuzzy Distance Based Approach' uses a very simple mathematics and is efficient enough to solve the problems which involve a large number of mutually interacting and dependent selection criteria. The proposed methodology is 
validated comparing the results with the results obtained using a well-known AHP technique. The comparison of the results shows that the proposed FDBA method is in good agreement with the AHP technique with an additional advantage of easy, simple reduced mathematical calculations. In order to validate the proposed approach, the results so obtained can be compared with such other techniques such as TOPSIS, PROMETHEE, Matrix or Fuzzy Matrix methods.

\section{References}

Alves, C., \& Castro, J. (2001, October). CRE: A systematic method for COTS components selection. In XV Brazilian Symposium on Software Engineering (SBES). Rio de Janeiro, Brazil.

Phillips, B. C., \& Polen, S. M. (2002). Add decision analysis to your COTS selection process. Software Technology Support Center Crosstalk.

Crnkovic, I., \& Larsson, M. P. H. (2002). Building reliable component-based software systems. Artech House.

Kunda, D. (2003). STACE: social technical approach to COTS software evaluation. In ComponentBased Software Quality (pp. 64-84). Springer Berlin Heidelberg.

Beus-Dukic, L., \& Bøegh, J. (2003). COTS software quality evaluation. InCOTS-Based Software Systems (pp. 72-80). Springer Berlin Heidelberg.

Erdogmus, H. (2001). Management of License Cost Uncertainty in COTS-Based Software Development: A Real Options Approach.

Faridi, M. S., Javed, Z., Abid, M. H., Ahmed, M., \& Ngadi, M. A. B. (2013). IROTS: A Proposed COTS Evaluation \& Selection Methodology for Component Based Software Engineering in UnderDevelopment Countries.

Faundes, M. J., Astudillo, H., \& Hitpass, B. (2013, September). Process-based evaluation and comparison of OTS software alternatives. In Computer Science and Information Systems (FedCSIS), 2013 Federated Conference on (pp. 1093-1100). IEEE.

Finkelstein, A., Spanoudakis, G., \& Ryan, M. (1996, March). Software package requirements and procurement. In Software Specification and Design, 1996., Proceedings of the 8th International Workshop on (pp. 141-145). IEEE.

Gaedke, M., Rehse, J., \& Graef, G. (1999, May). A repository to facilitate reuse in component-based web engineering. In International Workshop on Web Engineering at the 8th International WorldWide Web Conference (WWW8). Toronto, Ontario, Canada.

Gashi, I., Popov, P., \& Stankovic, V. (2009). Uncertainty explicit assessment of off-the-shelf software: A Bayesian approach. Information and Software Technology, 51(2), 497-511.

Gesellensetter, L., \& Glesner, S. (2007). Only the best can make it: Optimal component selection. Electronic Notes in Theoretical Computer Science,176(2), 105-124.

Haghpanah, N., Moaven, S., Habibi, J., Kargar, M., \& Yeganeh, S. H. (2007, December). Approximation algorithms for software component selection problem. In Software Engineering Conference, 2007. APSEC 2007. 14th Asia-Pacific (pp. 159-166). IEEE.

Ibrahim, H., Far, B. H., \& Eberlein, A. (2007, April). On Agent-Oriented Requirements Engineering for COTS-Based Software Development (CBSD). In Electrical and Computer Engineering, 2007. CCECE 2007. Canadian Conference on (pp. 690-693). IEEE.

Ibrahim, H., Far, B. H., \& Eberlein, A. (2008, November). Towards Uncertainty Management in COTSBased Development. In Proc. of the 9th IASTED International Conference on Software Engineering and Applications SEA (pp. 16-18).

Ibrahim, H., Far, B. H., \& Eberlein, A. (2009). Weighted criteria hybrid model for ranking commercial off-the-shelf products. In Proc. of the IASTED Int. Conf. on Software Engineering SE 2009 (pp. 4956).

Kaur, K., \& Singh, H. (2014). Quantifying COTS Components Selection using Multi Criteria Decision Analysis Method-PROMETHEE. Global Journal of Computer Science and Technology, 14(2).

Khan, A., Khan, K., Amir, M., \& Khan, M. N. A. (2014). A Component-Based Framework for Software Reusability. International Journal of Software Engineering and Its Applications, 8(10), 13-24.

Kontio, J. (1995). OTSO: a systematic process for reusable software component selection. 
Kontio, J. (1996, March). A case study in applying a systematic method for COTS selection. In Software Engineering, 1996., Proceedings of the 18th International Conference on (pp. 201-209). IEEE.

Kumar, R., \& Garg, R. K. (2010). Optimal selection of robots by using distance based approach method. Robotics and Computer-Integrated Manufacturing, 26(5), 500-506.

Mancebo, E., \& Andrews, A. (2005, March). A strategy for selecting multiple components. In Proceedings of the 2005 ACM symposium on Applied computing (pp. 1505-1510). ACM.

Mead, W. J. (2008). Uncertainty in Decision Making. Encyclopedia of Public Administration and Public Policy,

Mittal, S., \& Bhatia, P. K. (2013). Framework for Evaluating and Ranking the Reusability of COTS Components based upon Analytical Hierarchy Process.

Mohamed, A., Ruhe, G., \& Eberlein, A. (2005, May). Decision support for customization of the COTS selection process. In ACM SIGSOFT Software Engineering Notes (Vol. 30, No. 4, pp. 1-4). ACM.

Mohamed, A., Ruhe, G., \& Eberlein, A. (2007, March). COTS selection: past, present, and future. In Engineering of Computer-Based Systems, 2007. ECBS'07. 14th Annual IEEE International Conference and Workshops on the(pp. 103-114). IEEE.

Mohamed, A., Wanyama, T., Ruhe, G., Eberlein, A., \& Far, B. (2004). COTS evaluation supported by knowledge bases. In Advances in Learning Software Organizations (pp. 43-54). Springer Berlin Heidelberg.

Morisio, M., Seaman, C. B., Basili, V. R., Parra, A. T., Kraft, S. E., \& Condon, S. E. (2002). COTSbased software development: Processes and open issues. Journal of Systems and Software, 61(3), 189-199.

Morisio, M., Seaman, C. B., Parra, A. T., Basili, V. R., Kraft, S. E., \& Condon, S. E. (2000, June). Investigating and improving a COTS-based software development. In Proceedings of the 22nd international conference on Software engineering (pp. 32-41). ACM.

Nazir, S., Anwar, S., Khan, S. A., Shahzad, S., Ali, M., Amin, R., ... \& Cosmas, J. (2014, January). Software component selection based on quality criteria using the analytic network process. In Abstract and Applied Analysis. Hindawi Publishing Corporation.

Ncube, C., \& Dean, J. (2002). The Limitations of Current Decision-Making Techniques in the Procurement of COTS Software Components.

Ncube, C., \& Maiden, N. A. (1999). Guiding parallel requirements acquisition and COTS software selection. In Requirements Engineering, 1999. Proceedings. IEEE International Symposium on (pp. 133-140). IEEE.

Ruhe, G. (2003). Intelligent support for selection of COTS products. In Web, Web-Services, and Database Systems (pp. 34-45). Springer Berlin Heidelberg.

Rus, I., Seaman, C., Lindvall, M., Basili, V., \& Boehm, B. (2001). A Web Repository of Lessons Learned from COTS-Based Software Development1.Notes.

Saltelli, A., Ratto, M., Andres, T., Campolongo, F., Cariboni, J., Gatelli, D., ... \& Tarantola, S. (2008). Global sensitivity analysis: the primer. John Wiley \& Sons.

Tran, V., Liu, D. B., \& Hummel, B. (1997, July). Component-based systems development: challenges and lessons learned. In Software Technology and Engineering Practice, 1997. Proceedings., Eighth IEEE International Workshop on [incorporating Computer Aided Software Engineering] (pp. 452462). IEEE.

Vinay, M. K., \& Johri, P. (2014). W-Shaped Framework for Component Selection and Product Development Process. World Applied Sciences Journal, 31(4), 606-614.

Wong, B., \& Jeffery, R. (2002). A framework for software quality evaluation. In Product Focused Software Process Improvement (pp. 103-118). Springer Berlin Heidelberg.

Ziv, H., Richardson, D., \& Klösch, R. (1997). The uncertainty principle in software engineering. In submitted to Proceedings of the 19th International Conference on Software Engineering (ICSE'97). 